\title{
Detection of SARS-CoV-2 viremia before onset of COVID-19 symptoms in an allo-transplanted patient with acute leukemia
}

\author{
Veronica Di Cristanziano ${ }^{1} \cdot$ Charlotte Meyer-Schwickerath ${ }^{2} \cdot$ Kirsten Alexandra Eberhardt $\mathbb{C}^{3} \cdot$ Jan Rybniker ${ }^{2,4,5}$. \\ Eva Heger $^{1}$ - Elena Knops ${ }^{1} \cdot$ Michael Hallek ${ }^{2,6} \cdot$ Florian Klein $\mathbb{D}^{1,4,5} \cdot$ Udo Holtick $^{2,6} \cdot$ Norma Jung $^{2}$
}

Received: 15 June 2020 / Revised: 3 September 2020 / Accepted: 7 September 2020 / Published online: 18 September 2020

(c) The Author(s), under exclusive licence to Springer Nature Limited 2020

\section{To the Editor:}

COVID-19 is a life threatening disease, caused by infection with severe acute respiratory syndrome coronavirus 2 (SARS-CoV-2) and patients with undergoing treatment for hematologic malignancies including hematopoietic stem cell transplantation are considered to be at particular risk for fatal outcomes $[1,2]$.

This report describes for the first time the detection of SARS-CoV-2 viremia prior to the onset of symptoms and diagnosis of COVID-19 in a 51-year-old patient with acute myeloid leukemia (AML) and relapse after allogeneic hematopoietic stem cell transplantation (allo-HSCT). Notably, we illustrate that SARS-CoV-2 viremia inversely

These authors contributed equally: Veronica Di Cristanziano, Charlotte Meyer-Schwickerath, Kirsten Alexandra Eberhardt, Udo Holtick, Norma Jung

Supplementary information The online version of this article (https:// doi.org/10.1038/s41409-020-01059-y) contains supplementary material, which is available to authorized users.

Veronica Di Cristanziano

veronica.di-cristanziano@uk-koeln.de

1 Institute of Virology, Faculty of Medicine and University Hospital of Cologne, University of Cologne, Cologne, Germany

2 Department I of Internal Medicine, Faculty of Medicine and University Hospital of Cologne, University of Cologne, Cologne, Germany

3 Department of Tropical Medicine, Bernhard Nocht Institute for Tropical Medicine and I. Department of Medicine, University Medical Center Hamburg-Eppendorf, Hamburg, Germany

4 German Center for Infection Research (DZIF), Partner Site BonnCologne, Cologne, Germany

5 Center for Molecular Medicine Cologne, University of Cologne, Cologne, Germany

6 Center of Integrated Oncology ABCD, University Hospital of Cologne, Cologne, Germany correlates with anti-SARS-CoV-2 antibody production, which predates negativity in respiratory samples for 4 weeks.

A 51-year-old female patient was admitted to the University Hospital of Cologne on March 9, 2020, with herpes zoster involving C8/Th1 and Enterococcus faecalis bloodstream infection in the context of a third relapse of a FLT-3 mutated AML after an allo-HSCT from her HLAidentical brother in 2017. At this point, she presented with CTC (Common Toxicity Criteria) grade IV neutropenia and thrombocytopenia. Antibiotic and antiviral treatment was initiated. The patient received Midostaurin and Gemtuzumab ozogamicin as treatment for the relapsed AML. Under this therapy, the general condition of the patient improved, with symptoms and inflammatory parameters retreating.

However, on March 21, 2020, 11 days after admission, the patient developed fever with temperatures up to $38.4{ }^{\circ} \mathrm{C}$ combined with a dry cough and fatigue. SARS-CoV-2 infection was confirmed in a PCR analysis of a pharyngeal swab on March 23, 2020. Laboratory results showed an increase in inflammatory parameters such as ferritin, Creactive protein (CRP) and interleukin (IL)-6 (Fig. 1). A computed tomography (CT) scan showed infiltrates characteristic of COVID-19 pneumonia (see Supplementary Fig. 1). The patient rapidly developed dyspnea, peripheral blood oxygen saturation decreased and non-invasive oxygen supplementation was required (Fig. 1). Following, IL-6 and ferritin levels increased fast correlating with clinical decline. Under the assumption of a hyperinflammatory syndrome, the patient received Tocilizumab twice in a 7 days interval due to not satisfactory recovery after the first dose. After anti-IL6 treatment, symptoms decreased as well as inflammatory parameters (Fig. 1). The patient recovered slowly in the following 6 weeks: fever subsided with the exception of a new catheter-associated soft-tissue infection after 6 weeks. Oxygen supplementation could be slowly reduced and a progressive decrease of inflammatory 

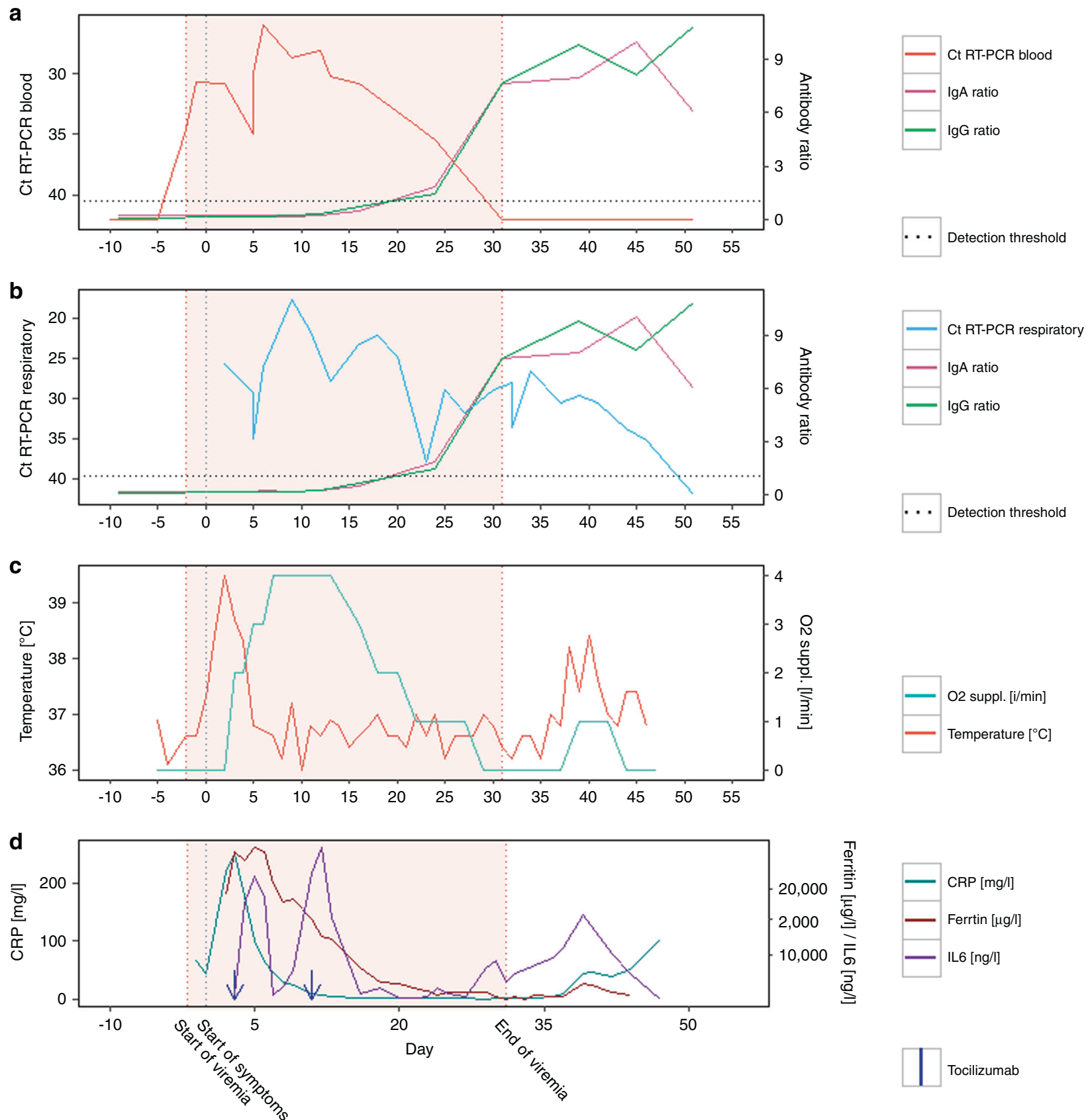

Fig. 1 Clinical and laboratory parameters related to COVID-19 in a 51-years-old acute leukemia patient over the observation period. a Real-time PCR Cycle threshold (RT-PCR Ct) values for severe acute respiratory syndrome coronavirus 2 (SARS-CoV-2) RNA in blood samples and IgG antibody ratios. The dotted horizontal line marks the detection line of RNA, as well as the threshold for antibody seroconversion. Onset of symptoms defined as day 0 , presented with grey vertical dotted line. Onset and end of viremia presented with red

parameters was observed. The patient was finally discharged seven weeks after admission and referred to our outpatient clinic for further treatment of her AML.

Respiratory and plasma specimens were collected routinely for SARS-CoV-2 real-time PCR (RT-PCR) and vertical dotted lines. Negative RT-PCR test results are displayed as Ct (Cycle threshold) values of 42. b RT-PCR Ct values for SARS-CoV-2 RNA in respiratory samples and corresponding $\operatorname{IgG}$ antibody ratios. c Maximum Temperature measured on each day in ${ }^{\circ} \mathrm{C}$ and maximum oxygen supply in $1 / \mathrm{min}$ on each day over the course of disease. Fever is defined as a temperature $>38.0^{\circ} \mathrm{C}$. d Levels of CRP (C-reactive protein) in $\mathrm{mg} / \mathrm{l}$, ferritin in $\mu \mathrm{g} / \mathrm{l}$ and interleukin (IL)-6 in $\mathrm{ng} / \mathrm{l}$ over the course of disease.

antibody testing (detailed information is reported in Supplementary Materials). The publication of this data was approved by the Institutional Review Board of the University of Cologne (20-1254) and the patient's written informed consent obtained. 
SARS-CoV-2 viremia was first detected on March 19, 2020, 2 days before the first symptoms (fever and dry cough) occurred and inflammatory parameters began to rise. Thus, we confirmed the presence of SARS-CoV-2 viremia 4 days before the detection of SARS-CoV-2 RNA in pharyngeal swab (Fig. 1).

Analyzing the virus-related molecular and serological parameters throughout the course of disease, the progressive increase of detected SARS-CoV-2 RNA in the evaluated plasma samples reached a peak 8 days after the first detection in blood, whereas the last positive evidence of SARS-CoV-2 RNA in plasma was observed on April 14, 2020. On the same date, corresponding to 24 days after initial symptoms, SARS-CoV-2 specific IgA and IgG antibodies were detected (above threshold of 1.1). Along the course, the increase of specific SARS-CoV-2 antibody levels correlated with viral clearance from the blood (Fig. 1). However, SARS-CoV-2 RT-PCR from respiratory samples remained positive for three more weeks ( 7 weeks in total), despite occurrence of seroconversion, disappearance of COVID-19 related symptoms, and normalized inflammatory markers. Finally, 51 days after the onset of symptoms, the patient was tested SARS-CoV-2 negative in respiratory specimen (Fig. 1).

The present report describes a case of SARS-CoV-2 viremia, preceding COVID-19 symptoms for 2 days, indicating a clear link between the observed SARS-CoV-2 viremia and beginning of COVID-19 symptoms. Consecutively, this suggests that SARS-CoV-2 viremia might play a more crucial role in COVID-19 than previously assumed because viremia before onset of symptoms is possible at least in a portion of cases. So far, SARS-CoV-2 RNA detected in blood has been considered a bystander effect, assuming that it is an avital virus component [3]. However, the detection of viremia prior to the onset of clinical symptoms suggests a systemic infection with SARS-CoV-2, which is supported by findings of detected virus components in other organs than the lung [4]. Based on these results, we hypothesize that the viremia plays a more relevant role in SARS-CoV-2 infection than in other respiratory viral infections due to lacking immunity in the population as demonstrated for pandemic influenza strains [5].

Notably, our results underline previously upcoming concerns about SARS-CoV-2 blood-borne transmission following blood donations and hematopoietic stem cell transplantation [6, 7]. Giving the possible systemic nature of the infection, a close evaluation of stem-cell donors as well as transplant candidates is needed as already proposed by the EBMT [8] since a systemic infection in highly immunocompromised individuals can lead to sepsis and consecutively fatal outcomes.
Our clinical case reports the observation of detectable SARS-CoV-2 antibody production later in the course of the disease than reported in other patients before, as most of the patients show a seroconversion 10-14 days after diagnosis [3, 9], which might be due to the patient's immunosuppressive state caused by the underlying acute leukemia. However, longer time periods until observation of seroconversion have been described before [10], which might be as well due to different serological assays used with varying sensitivity, based on the target viral antigens [11]. Nevertheless, in our case antibody detection clearly coincided with viral clearance in plasma and improvement of clinical symptoms, correlating with previous observations [12] and thus underlining the observed timeline.

However, as immunosuppression and underlying comorbidities are relevant risk factors for obtaining clinical relevant COVID-19 infections, the delayed occurrence of seroconversion has implications for individual subjects, as well as for public health systems with only limited resources of intermediate-care treatment capacities. Faster development of an antibody-response in immunocompetent patients might hinder or preclude detection of positive blood samples at the time of hospitalization.

In the clinical case presented here, the heavily pre-treated allo-transplanted leukemia patient fortunately survived the infection, showing that additional factors, including early diagnosis and prompt supportive therapy, can improve the outcome of COVID-19 in immunocompromised patients. It is unclear, if the application of Tocilizumab accounted for the cure of the patient. A severe cytokine storm in COVID19 is discussed as a cause of death with Tocilizumab as a treatment option to diminish the storm by blocking IL-6. Nevertheless, the overall certainty of evidence is still very low [8].

Concluding, based on our findings a systemic nature of SARS-CoV-2 is a relevant aspect during the early phase of infection and is correlated with specific antibody clearance, indicating a clear interaction between humoral immune response and control of SARS-CoV-2. Further, it shows that a continuous supportive treatment of allo-transplanted patients with COVID-19 can lead to a favorable outcome even in a situation with a heavily compromised immune status.

Acknowledgements We thank the patient described in this report for her support and wish her the best of health. Further, we would like to thank Clara Lehmann, Lukas P. Frenzel, Marco Herling, Bastian von Tresckow and Philip Posdzich for their support and clinical input in treating the patient, Carola Heneweer for providing the radiological images, and Rolf Kaiser for the support in obtaining and interpreting the RT-PCR and serological data. 
Conflict of interest The authors declare that they have no conflict of interest.

Publisher's note Springer Nature remains neutral with regard to jurisdictional claims in published maps and institutional affiliations.

\section{References}

1. Núñez-Torrón $\quad C, \quad$ García-Gutiérrez $\quad V, \quad$ Tenorio-Núñez MC, Moreno-Jiménez G, López-Jiménez FJ, Herrera-Puente P. Poor outcome in patients with acute leukemia on intensive chemotherapy and COVID-19. Bone Marrow Transplant. 2020. https://doi.org/10.1038/s41409-020-0961-y.

2. Malard F, Genthon A, Brissot E, van de Wyngaert Z, Marjanovic Z, Ikhlef S, et al. COVID-19 outcomes in patients with hematologic disease. Bone Marrow Transplant. 2020. https://doi.org/10. 1038/s41409-020-0931-4.

3. Wolfel R, Corman VM, Guggemos W, Seilmaier M, Zange S, Muller MA, et al. Virological assessment of hospitalized patients with COVID-2019. Nature. 2020;581:465-9.

4. Wang W, Xu Y, Gao R, Lu R, Han K, Wu G, et al. Detection of SARS-CoV-2 in different types of clinical specimens. JAMA. 2020;323:1843-4.

5. Likos AM, Kelvin DJ, Cameron CM, Rowe T, Kuehnert MJ, Norris PJ, et al. Influenza viremia and the potential for bloodborne transmission. Transfusion. 2007;47:1080-8.
6. Chang L, Zhao L, Gong H, Wang L, Wang L. Severe acute respiratory syndrome coronavirus 2 RNA detected in blood donations. Emerg Infect Dis. 2020;26:1631-3.

7. Anurathapan U, Apiwattanakul N, Pakakasama S, Pongphitcha P, Thitithanyanont A, Pasomsub E, et al. Hematopoietic stem cell transplantation from an infected SARS-CoV2 donor sibling. Bone Marrow Transplant. 2020. https://doi.org/10.1038/s41409-0200969-3.

8. @ TheEBMT ESfBaMT. BMT. Coronavirus disease COVID-19: EBMT recommendations. 2020. https://www.ebmt.org/ebmt/ documents/coronavirus-disease-covid-19-ebmt-recommendationsupdate-may-18-2020.

9. Xiang F, Wang X, He X, Peng Z, Yang B, Zhang J, et al. Antibody detection and dynamic characteristics in patients with COVID-19. Clin Infect Dis. 2020. https://doi.org/10.1093/cid/ciaa461.

10. Long QX, Liu BZ, Deng HJ, Wu GC, Deng K, Chen YK, et al. Antibody responses to SARS-CoV-2 in patients with COVID-19. Nat Med. 2020;26:845-8.

11. Weidner L, Gänsdorfer $\mathrm{S}$, Unterweger $\mathrm{S}$, Weseslindtner $\mathrm{L}$, Drexler C, Farcet M, et al. Quantification of SARS-CoV-2 antibodies with eight commercially available immunoassays. J Clin Virol. 2020;129:104540.

12. To KK-W, Tsang OT-Y, Leung W-S, Tam AR, Wu T-C, Lung $\mathrm{DC}$, et al. Temporal profiles of viral load in posterior oropharyngeal saliva samples and serum antibody responses during infection by SARS-CoV-2: an observational cohort study. Lancet Infect Dis. 2020;20:565-74. 